\title{
Risk and Responsibility
}

Author: William Leiss, Christina Chociolko

Publisher: McGill-Queen's University Press (1994-07-01)

Buy this book from $\underline{\text { Amazon }}$ or Chapters.

Bitter disagreements arise over how to manage health and environmental risks. Trying to determine what is in the public interest is at the heart of these disagreements, but the core concerns of major sectors - industry, governments, and voluntary associations - are also at stake. Attempts to defuse the controversies and find solutions acceptable to all parties have met with little success.

Leiss and Chociolko show that controversies arise in part because many participants try to avoid assuming full responsibility for the consequences for the risk taking they advocate. Through documented case studios they address the difficulties of arriving at reliable scientific estimates of risk in controversial areas and the impact of this uncertainty on disagreements among different interest groups over how to manage those risks responsibly. In conclusion, they attempt to delineate conditions under which consensus on the assessment and management of environmental health risks might be achieved among a wide range of interest groups.

Tables and Figures

Acknowledgments

PART ONE: The Roots of Disagreement

PART TWO: Obstacles to Consesnsus: 
Two Seemingly Intractable Controversies

Power Frequency Electric and Magnetic Fields. Part One: Expert Assessment

Power Frequency Electric and Magnetic Fields. Part Two: A Public Controversy

Alar, or Who Benefits?

154

PART THREE: Towards Consensus

Stakeholder Negotiation

197

Antisapstain Chemicals

219

Useful Lessons

256

Appendix 1: PF E/MF and Human Health

Basic Concepts

Appendix 2: Antisapstain Chemicals Stakeholder Negotiation Documents

Notes

317

Bibliography

Index

399

\section{Index}

A

Acceptable daily intake (ADI), 34 
Acceptable risk, 22, 23, 27, 32-4, 38, 39, 40, 44, 50, 64, 203, 222, 229, 234, 253, 261, 262, 265

Accidents, risk from automobile, 7, 11, 56, 57, 65, 68, 259

Accutane, 45

Addiction, 74, 76, 329n.42

Administrative Conference of the United States, 211

Advertising, $71,72,75$

Advocacy groups. See Public interest groups

A. H. Robins Corporation, 53

Air pollution, 69

Alachlor, 16-23, 320-21, 321-323, 372-380

Alar (daminozide, SADH), 14, 15, 17, 22, 23, 154-93, 197-206, 266, 268, 274-6, 319n.15, 362n. 68

Alcohol, risk from, 7, 56, 57, 65, 66

Aldicarb, 179-80, 274

Allocative effects, 41

Alternating current (AC), 81,375n.4

American Council of Government and Industrial Hygienists, 372n.16

Ames, Bruce, 363n.70, 371n.14

Animal (laboratory) studies, 21,48, 55, 856, 89, 91-2, 111,114, 161-2, 163-5, 168, 184, 190, 297-9, 323n.16

Antisapstain chemicals, 15, 22, 206-7, 219-55, 259, 276-7

Appliances, and exposure to risk, 57, 65, 86, 101, 127, 133, 134, 287-8

Asbestos, 8, 10, 2o, 21, 53

'As low as reasonably achievable' (ALARA) guideline, 40, 50, 203

Azaconozole, 226, 237, 240

B 
Bacteria (moulds, fungi), and exposure to risk, 62, 219, 220, 266

Bailey, William, 107

Banks, Robert S., 150

Barker, Jeff, 107, 115, 132, 137

Bauman, R.J., 115, 120

Bawin and Adey, 92

Beavan, Pat, 105, 115, 132

Becker, Robert, 105, 111, 140, 149

Bell, Larry, 104

Benefit-Cost Analysis (BCA),41

Benefits, 40, 42, 44, 50, 51, 52, 54-9, 170-2, 226; types of, 60-5

Benzene, 20

"Best alternative to a negotiated agreement" (BATNA), 209

Bioaccumulation, 32, 160

Bioelectromagnetics Society, 94

"Black box" model, 172, 250, 253, 271

Boatman, Chris, 106, 107, 109, 115, 132

Bonneville Power Administration, 92, 101, 104, 148, 335n.46

Borax, 228, 307, 309, 312, 314

Bradley, Ed, 154, 160, 180

British Columbia; Commission on Resources and Environment, 212;

Fruit Growers Association, 178-9;

Hydro and Power Authority, 102-53;

Ministry of Environment, 225, 228, 245, 267, 303, 308, 312, 314;

Ministry of Forests, 234, 245, 305; 
Ministry of Health, 112, 115, 132, 135, 145, 267;

Public Interest Advocacy Centre, 105;

Roundtable on Environment and the Economy, 212;

Stakeholder Forum, 229-48, 277, 303-8, 367n.1;

Tree Fruits Ltd., 179;

Utilities Commission, 102, 105, 111-13, 115-36, 144, 146

Brodeur, Paul, 113

Brunk, Haworth, and Lee, 18, 19, 22, 23, 372n.20

Bush, George, 30; Bush administration, 55

C

Cadmium, 20, 54-8

Calcium, 84, 92, 331n.9

California Department of Food and Agriculture, 191

California EMF Consensus Group,272

Canada: Agriculture Canada,62, 183, 199, 220, 226-9, 231, 233-4, 241, 244, 246-7, 248, 251, 277, 303-15;

Environment Canada, 199, 224, 225, 226, 227; Forestry Canada, 234;

Fisheries and Oceans Canada, 224, 226, 227;

Health and Welfare Canada 18, 19, 97, 155, 158, 161, 162, 163, 165, 183-4, 189-90, 224, 226, $247,248,251,275,276$

Canadian Association of Pesticide Control Officers (CAPCO), 246, 309-15

Canadian Electrical Association, 132, 136

Canadian Environmental Protection Act, 211

Canadian Nuclear Association, 70-3

Canadian Pacific Forest Products, 102-3

Canadian Paperworkers Union, 225, 245 
Canadian Particle Board Association, 50

Cancer, 9, 31, 40, 55, 60, 126, 178, 332n.15;

childhood, 86-7, 96, 104, 106,

$108,111,117,130,154,155,165,189,338$ n.8o, 343n.16, 375n.34; classification of, 17, 147, 166-7, 190, 191;

and daminozide and UDMH, 161-8, 177, 188-92;

and EMF, 84, 86-8, 93, 96, 119, 121, 124;

potency of, 167, 168;

and smoking, 74 .

See also Carcinogenesis

Capitalism, and incentive to off-load risk, 52

Captan, 374n.32

Carcinogenesis, 19, 48-9, 73, 86, 164, 166, 177

Carnegie Mellon University, 94, 101, 133

Carson, Rachel, 53

Cause-and-effect relationships, 53, 54, 126,258, 299, 301-2

CBS Television, 154, 156

Cellular structure, effects of EMF exposure on, 84-5, 97, 117, 298

Central nervous system, effects Of EMF exposure on, 85-6, 96

Chemicals (chemical industry), 20, 22, 27, 31,61,62, 65 , 148, 201-2, 223, 231-2, 374n.22

Chlorine, 15, 62, 32on.16

Chlorophenates, 220-2, 227, 231, 251, 309

Circadian systems, and ELFexperiments, 86, 331n.12

Clorox Co., 182

Coberly, Robert, 161 
Code of Good Practice, 244

Compensation, $21,41,51,56,58,62,104,105,113,203$

Congress (U.S.), 266

Consensus, 38, 39, 151 , 156, 159, 186, 198-2o6, 207-8, 212-15, 217-18, 229, 230-3 , 242, 246, $253,259,262-71,277-9,364 n .8$

Consent to risk-taking activity, 256-7

Consumer interests, 202-6

Consumers Association of Canada, 184

Consumers Union (U.S.), 184-5

Copper-8, 226, 228, 235, 236, 240, 307, 309, 312, 314

Corona, 90,333 n.27

Coronary heart disease, and tobacco use, 74

Cost-Effectiveness Analysis, 40, 44

Council of Forest Industries of B. C., 225, 228, 241, 245, 303, 307, 310

Courts, 14, 17, 18, 20, 52, 54, 99, 141, 151, 156, 258, 269, 319n.15, 336n.61, 340n.101, 352n. 157,372 n.21

Covello, Vincent, 35

Curley, Ken, 107, 110

"Cybernetic paresis," 267

D

Dalkon Shield, 53

Daminozide. See Alar DDAC, 226, 233, 236, 240, 241, 244, 307, 312, 314

DDT, 22, 53

Decision-making, 42, 47, 64, 310-15

Depression, and ELF experiments, 86 
Diet, and risk-taking, 57, 65, 266

Diethylstilbestrol, 4

Dioxins, 20,33, 221, 309, 361n-48

Direct current (DC), 375n.4

"Discussion Document on Anti-Sapstain Chemicals," 226-9, 234, 236, 251, 308, 310

Dispute resolution, 211, 364n.13

DNA, $49,84,331 \mathrm{n} .10$

Dose-Response Assessment, 29, 48, 49, 98, 147, 167

Driving, See Accidents, risk from automobile

Drugs, risk from, 20,34, 56, 65, 86; prescription, 42, 44-5, 59,60, 252, 257, 278

$\mathbf{E}$

Earthcare, 245, 312

Ecobrite, 314

Edwards, Ann, 115

Electric blankets, risk from, 87, 93, 98, 101, 114, 119, 123, 131, 133

Electric fields, 283, 285-6,289-90

Electric Power Research Institute, 91, 92, 100, 101, 125, 126, 148

Electricitie de France, 98

Electricity, $15,89,148,283$

Electromagnetic spectrum, 283-5

Energy sources, risk from, 59, 66, 70, 73

Environment, risks to, 20, 62, 260

Environmental Protection Agency (u.s.), 18, 22, 44, 45, 92, 147, 154-93, 198, 199, 211, 266,

268-9, 274, 326n-5; Health Effects Division Peer Review Panel, 166, 190, 192, 268-9; Scientific Advisory Panel, 18, 166, 172-3, 177, 190, 200, 269, 320n.21, 321n.23, 359n.37 
Environmental Research Information Inc., 1o8-9, 117, 128

Environmental Resources Group,'75

Environmental toxicity, 237-9

Epidemiology, 28, 48, 53, 83, 86, 94, 98, 114, 118, 122, 243, 247, 267, 299-301, 379n.72

Erdreich, Linda, 114, 115, 116ff., 125, 138, 139, 140, 146, 348n.73

Ethylene dibromide (EDB), 168, 179, 200, 267, 274, 371n.14

Experts, and risk management, 31, 32, 35-9, 534, 55-6, 58-60, 63, 66, 68, 70, 71, 137-8, 140-3, 157, 158, 204-5, 263, 267;

expert disagreement, 71, 97, 112, 114-15, 169-75;

expert testimony, 115-31

Exposure, 7, 27, 28, 31, 44, 46;

to antisapstains, 227, 313;

to daminozide and UDMH, 167-8, 174;

to $\mathrm{EMF}, 86,99,123,139,291-7$;

measurement of, 18, 29, 49, 93, 96, 98, 118, 121, 32in.27;

Occupational, 21,50, 54, 61 .

See also Risk, occupational

Extrapolation, from studies to human health effects, 21, 29, 37, 55, 167, 201

"Extremely low frequency," (ELF) fields, 84

\section{$\mathbf{F}$}

F2, 233, 236

Faraday, Michael, 88-9

Farmworkers. See Risk, occupational

Federal Insecticide Fungicide and Rodenticide Act (U.S.), 322n.7

Feldspar, 8

Fenton Communications, 181-2 
Firearms, risks from, 57

Fisher and Ury, 209

Food, risks from, 34, 59, 62, 66, 202, 204, 221, 257, 260, 266

Food and Drugs Act, 76, 26o

Forest Industry Industrial Health Research Program, 225

Forintek, 225, 236, 237

Formaldehyde, 20, 42, 48-50, 54, 64, 252, 259, 266, 319n.15, 325n.42

Framework Consensus Agreement, 241-6;text of, 303-8

Friends of the Earth, 184

Frum, Barbara, 183

G

Gallagher, Richard, 114, 115, 11,138, 139, 140, 145, 146

Gathercole, Richard, 105, 106, 107, 110, 113, 115, 116-22, 124

Genotoxicity, 48, 49

Gerber Products, 177

Gilbert, Phil, 241, 244

Glutamate Association, 182

Governments: role of in negotiation, 207-8, 212-13,278;

role of in risk communication, 37 ;

role of in risk management, $22,23,27,33,39,40,49,50,51,53,54,55,57,69,72,70,207-8$, 212-13, 259-61

Graham, Charles, 117

Grant, Bill, 112, 115

Graves, H.B., 125

"Greenhouse effect," 70 
Greenpeace, $182,320 \mathrm{n} .16$

Gregory, Robin, 319n.8

Gustafson, Carl, 112, 115, 117, 118, 119, 122, 128-30

H

Harrison and Hoberg, 157

Harter, Philip, 211

Hathaway, Janet, 197

Hazardous Products Act, 49, 76, 260

Hazards, 28-33, 61;

hazard warnings, 75 ;

hazardous waste, 10 ;

industrial, 61-2; natural, 20, 28, 57, 59, 60, 278

Health care costs, $11,57,329 \mathrm{n} .42$

Health Monitoring Subcommittee, 245-7, 306-7

Hertz, Heinrich, 88-9

Hertzman, Clyde, 241, 242, 243

Hill, Sir Austin Bradford, 302

Houston Lighting and Power, 94, 99

Human experimentation, 121, 122, 124

Hydrazines, 162, 371n12

Hydro Quebec, 98

\section{I}

Immune response, and ELF experiments, 84

Induction, 289-90 
Industrial Bio-Test Laboratories, 17, 53

Infectious disease control, risk involved in, 61,62, 278

Insurance, and risk assessment, 57, 58

Interest groups. See Public interest groups

International Agency for Research on Cancer, 48, 163, 166

International Apple Institute, 185, 189,197

International Radiation Protection Association, 83, 95

IPBC,226, 233, 240, 244, 307, 312, 314

IWA-Canada, 225, 241, 245, 247

\section{$\mathbf{J}$}

Jasanoff, Sheila, 150, 158

Johns-Manville Corporation, 53

$\mathbf{K}$

Kantrowicz, Arthur, 149

Kavka, Darlene and Frank, 105-10, 113, 114, 115

Ketcham Communications, 182

Kiln drying, as treatment for sapstain fungi, 219-20, 235, 244

Kirkpatrick, Diane, 183-4

Klassen, Frank, 104

Kop-Coat Inc., 236, 237, 240, 314

Koren, Herman, 189

Krewski, Dan, 42; Krewski and Birkwood, 46

$\mathbf{L}$

Lapsley, Paul, 168 
Lasso, 17

Lave, Lester, 178

Lead, 8, 20, 373n.21

League of Women Voters, 70

Liability, 5, 141, 256

Loss, 4, 8, 9, 12, 16,28, 44, 51-4, 56-8, 66

"Low frequency" fields, 84

Lumber Anti-Sapstain Advisory Committee, 225, 232, 235

Lung disease, and tobacco use, 53

M

MacCrimmon, Kenneth, 11, 12,28

McIntyre, John, 112,115, 132, 139

MacKenzie, Ken, 114, 115, 123, 125-7

MacMillan Bloedel, 242, 243, 242, 277

McMullan, Peter, 109

Magnetic fields, 82, 87, 93, 95, 96, 118, 119, 124, 133, 134, 283, 286-8, 289-90

Magnetophospenes, 82

Mammalian toxicity, 237-8

March, Lorne, 104, 107

Marino, Andrew, 105, 110-11, 112, 114-17, 123-31, 116, 138-40, 145, 146, 149, 150

Martin, Hu, 242, 243

Marton, John, 104, 1051, 107, 110, 123, 139, 145-6

Maximum Residue Limit 161, 165, 183, 190

Maximum Tolerated 191, 363n.70

Mazur, Allan, 149-50 
Media, role of in risk communication, 35, 37, 46, 71, 83, 88, 99, 122, 155, 181, 275

Medical procedures, risks from, 60-1, 74

Mercury, 8, 366n.19

Methane, 54, 259

Monosodium glutamate, 182

Monsanto Corporation, 17, 18,19, 321n.23, 372n.20

Moore, Jack, 154, 176, 177, 180, 180

Mott's, 177

Mutagenicity, 84, 164, 165, 191, 240,362n.68

$\mathbf{N}$

Nabisco, 177

Nader, Ralph, 175, 275

Napier Pacific Industries, 236, 237, 314-15

National Coal Policy Project, 211

Natural Resources Defense Council (NRDC), 154, 156, 157, 164, 169, 171, 174, 177, 180 , 181-2, 189, 191, 197, 266, 274, 275, 370n-9, 375n-33, 34; Intolerable Risk, 192-3, 204

Negotiation, 16,48, 207-9, 214, 215, 217-18, 243, 249-53, 262-71;

efficiency in, 216,273;

facilitation in, 209;

fairness in, 215, 273;

mediation in, 209;

and negotiated rule-making, 211,365n.14;

stability in, 217, 273;

wisdom in, 216, 273

Newsome, Robert, 163 
New York Power Authority, 91

New York State Power Line Project, 86, 96, 97, 104, 138, 150

New York State Public Service Commission, 91, 108, 111

New York Times, 175

Niagara Institute, 211

Nichols Dezenhall Communications, 182

Nicholson, William J., 193

No-effect level (NOEL), 33

Non-allocative effects, 41

Non-human nature, 60, 61,63, 370n.10

Non-ionizing radiation, 95, 100, 273, 283-5

"Not in my back yard" (NIMBY) syndrome, 261

NP-1, 233, 236, 240, 242, 243, 244, 245, 314

Nuclear power, 20,46, 62, 65, 66, 70, 71, 73, 328n.31

$\mathbf{O}$

Occupational Health and Safety Administration, 55, 211

Ontario Hydro, 97, 98, 109

Organochlorines, 221

Ozawa and Susskind, 271, 272

\section{$\mathbf{P}$}

Pentachlorophenol, 220, 221, 227, 228, 231, 251, 310, 313, 367n-3, 369n.20

Perception of risk. See Risk perception

Pest Control Products Act, 309, 313, 368n.18

Pesticides, 8, 20,40, 41 , 42, 59, 62, 66, 71, 160, 179, 202, 203, 220, 244, 257, 266, 267, 309-15, 322n.7 
Probability, 28-32, 37, 40, 47, 378n.60

Project Sanguine, 91, 333n.35

"Prudent avoidance ‘ Policy, 95, 101, 148-9

Psychometric research, 10

Public interest groups, 35, 36, 39, 44, 58, 200-6, 262, 264, 266, 267, 275, 278

Pulp, Paper and Woodworkers of Canada, 225, 245, 312

Q

Quaternary ammonium compounds, 241

$\mathbf{R}$

Radiation, 31, 62

Radio frequency waves, 92

Radon, 8, 6o, 69

Rayner, Steve, 256

Reagan administration, and deregulation, 55, 164, 274

Recreation, risks involved in, 56, 57, 65, 68

Regulation: decisions and process, 33, 41-5, 48, 83, 143, 211, 221-3, 226, 227, 244-5, 248, 249-50, 270-1, 309-15; role of government agencies in, 22, 23, 28, 34, 36, 39, 55, 88, 122, 183, 220,277

Regulatory negotiation, 211-12, 364n.8, 365n.16,370n.8, 9

Reserve Mining, 319n.15

Residential wiring, and exposure to magnetic fields, 93, 96, 127, 134, 337n.77

Risk: concept of, 3, 6, 28;

distribution of, $33,34,62,64,210,278$;

excess, $9,49,56,62,203,259,269,323 n .26,374$ n.22;

involuntary, 13, 27, 51, 56, 58, 68, 69, 73, 204, 257, 259, 262, 265, 278; 
ranking of, 70; r

eduction of, 270, 275;

responsibility for, 4-6, 51-4, 258, 265-71, 273-4, 277;

types of, 60-5;

voluntary, 20, 27, 31, 56, 66, 68, 73, 204, 257, 265, 278.

See also Acceptable risk; and the following entries

Risk assessment (risk analysis, risk estimation), 7, 20-1 ,28, 29-30, 32, 35, 37, 38-9, 41, 42, 46-8, 57,144-6, 263-5, 364n.7, 373n.21, 375n.33;

and Alachlor, 18; and antisapstains, 226, 227, 251, 252;

and daminozide and UDMH, 161-6, 169, 173, 183-4, 188-93, 268-70;

and EMF, 91, 95, 108-9, 133-4, 147-8;

and formaldehyde, 48-9;

and intuitive judgment, $8,30,38,58,59,65,68,70,71,72$;

and pathology, 19, 21, 167;

and qualitative judgment, 39;

and smoking, 74;

and uncertainty, 7, 9, 30, 46, 47, 52, 137, 139, 142-4, 277, 279, 319n.8, 353n.171;

and under-assessment, 10, 52-4, 258, 259, 260

Risk/benefit analysis, 18, 40, 170-4, 187, 191, 324n.34

Risk/benefit trade-offs, 19, 23, 33, 34, 39, 42, 44, 45, 50, 51, 56-73, 58-60, 171, 203, 204, 210, $250-1,253,256-7,270,277$

Risk communication, 348, 46, 65, 69, 73, 101, 198, 354n.189

Risk comparisons, 33, 34, 71, 74, 233-5

Risk constituencies, 259-62

Risk management, 27-50, 58, 262-71, 309-15

Risk, occupational, 59, 61-2, 77, 326n.10, 374n.22; 
and antisapstains, 221,233, 242, 249, 259, 260, 261, 266;

and cadmium, 545;

and daminozide and UDMH, 202,203;

and EDB,371n.14;

and EMF, 86-8, 90, 93, 97-9, 114, 118;

and farmworkers, 200-1, 203, 206, 266, 327n.14, 371n.15;

and formaidehyde, 50, 54, 64, 259;

and mining, 54, 61-2, 257;

and tobacco, 330n-46

Risk perception, 21,30-2, 37, 46, 47, 59, 65, 66, 73, 104, 146, 176, 179

Risk-averse behaviour 4, 10, 16, 20,57, 157, 262, 264, 265, 266, 267, 268, 270

Risk-promoters, 259, 264, 268-71,373n.21

Risk-taking behaviour, 4, 91 11-16, 20,52, 54, 58

RNA, and ELF experiments, 84, 331n.10

Robertson, Herb, 131

Ross, Randy, 115

Royal Society of Canada, 73, 329n.42

$\mathbf{S}$

SADH.See Alar

Safety factors, 33, 162, 189, 192

Safeway, 175, 178

Salter, Liora, 140-4

Sandborn, Calvin, 240, 242

Sandman, Peter, 69, 198

Sastre, Antonio, 114, 115, 116ff., 125, 138, 139, 140. 146, 349n.73 
Savitz, David, 96, 97, 104, 108, 117, 119, 126, 127, 138, 140

Schiefer, H.B., 236-43

Science, 21-2,29, 38, 40, 54, 55, 58, 116-17, 119-20, 125-7, 138-44, 146, 155; and EMF, 90-2,127; "mandated science," 17, 140-4, 374n.31; and peer review, 38, 39, 150, 201; and Policy, 45-7, 140-4; and research funding, 120,124-7, 130-1; science court, 149-50; scientific audit, 167,268, 359n.36; scientific consensus finding conference, 151, 272, 273; scientific controversy, 90-1, 97, 99, 119-23, 129-30, 167, 172-3, 201, 271-2; scientific panels, 150

Sexually transmitted diseases, risks of, 56-7

Shannon, Elizabeth, 107, 110

Shrader-Frechette, K. S., 9, 323n.26

"6o Minutes," 154, 180, 181, 182, 183, 197

Skelly, Bob, 115

Slovic, Paul, 10, 27, 32, 66, 70

Smith, Neptune, 112, 115

Smoking. See Tobacco

Socio-Economic Impact Analysis, 41

Solomon, K.R., 236-43

Somers, Emmanuel, 42

Stakeholders, 206-8, 229-55, 259-65, 272, 370n.10

Streep, Meryl, 180, 181

Susskind and Cruikshank, 213, 215-16, 273

Suzuki, David, 137

Swanson, Milt, 112,115

Sweden, 48

$\mathbf{T}$

TCMTB ,225, 226, 228, 231, 235, 236, 240, 307, 309, 312, 314 
Tesla, Nikola, 88-9

Thate, Clifford, 131

Thompson, Thom, 106, 107

"Threshold limit value," 50, 372n.16

Timbercote, 233, 236, 314

Tobacco, 5, 7, 8, 11, 27, 48, 50, 53, 54, 56, 57, 59, 66, 69, 71, 73-7, 124, 240, 252, 259, 325n.4, 326n-5, 328n.41, 329n.42, 330n-46, 370n-3, 371n12

Toth, Bela, 161-9,172-3, 190, 191, 200, 201, 268-9, 274, 371n.12

Toxic chemicals. See Chemicals

Toxicology, 18, 28, 32, 42, 55, 118, 161, 169, 269

Trade-offs. See Risk/benefit trade-offs

$\mathbf{U}$

UDMH, 157, 161-8,173, 176, 183, 188-93, 266, 274, 356n.1, 362n.68, 371n.12, 372n.16

Unions, $185,224,226,227,235,250,251,261,264,276,370 \mathrm{n}-7$

Uniroyal Chemical Co., 154-75, 177, 182, 199-206, 268-9, 274-6, 319n.15, 372n.19, 21

United Kingdom: Ministry of Agriculture, Fisheries and Food, 192; National Radiological Protection Board, 147

United States: Department of Agriculture, 174, 181, 199;Department of Energy, 91, 92, 94, 96, 97, 100, 101; Environmental Protection Agency, 18, 22,44, 45, 92, 147, 154-93, 198,199, 200, 211, 266, 268-9, 274, 326n.5; Food and Drug Administration, 181, 199; National Academy of Sciences, 91; National Cancer Institute, 98, 123, 163; National Environmental Protection Act, 89, 91; National Institutes of Health, 124;National Research Council, 91, 150; National Science Foundation, 29; Office of Technology Assessment, 133, 147; Rural Electrification Administration, 88

Upper-bound (95 percent) confidence limit, 265-6, 358n.28, 379n-71

Uranium, 8,70

Urea formaldehyde foam insulation (UFFI), 48, 48, 50, 266, 373n.21

User groups, role ofin negotiation, 200-6 
Vance, Joan, 115, 132

Vander Zalm, Bill, 103

Vigod, Toby, 184

Vinyl chloride, $8,321 \mathrm{n} .27$

Viscusi, Kip, 329n.42, 369n.2

Volta, Alessandro, 88-9

W

Walsh, Karen, 113, 114

Ward, Hans, 240

Warner, Kenneth E., 76

Washington (State of), 156, 172, 176, 177, 273

Wehrung, Donald, 11, 12, 28

Wertheimer and Leeper, 87, 93, 96, 97, 108, 113, 119, 126,335n.52

Wessel, Milton, 151

West Coast Environmental Law Association, 240, 245, 312

Westray mine, 54, 259

Windowing, in cellular structure, 84, 92, 93, 294

Wong, Paul, 115, 123, 132

Workers, Workplace. See Occupational risk

World Health Organization, 83, 95, 108, 127

Wynne, Brian, 51

$\mathbf{Z}$

Zero risk, 51, 264, 265, 267 